\title{
Development of a Hospital-Based Program Focused on Improving Healthcare Value
}

\author{
Christopher Moriates, MD*, Michelle Mourad, MD, Maria Novelero, MPA, Robert M. Wachter, MD
}

Division of Hospital Medicine, University of California, San Francisco, San Francisco, California.

BACKGROUND: Frontline physicians face increasing pressure to improve the quality of care they deliver while simultaneously decreasing healthcare costs. Although hospitals and physicians are beginning to implement initiatives targeting this new goal of healthcare value, few of them have a well-developed infrastructure to support this work.

METHODS: In March 2012, we launched a high-value care (HVC) program within the Division of Hospital Medicine at the University of California, San Francisco. The HVC program is co-led by a physician and the division's administrator, and includes other hospitalists, resident physicians, pharmacists, and administrators. The program aims to (1) use financial and clinical data to identify areas with clear evidence of waste in the hospital, (2) promote evidence- based interventions that improve both quality of care and value, and (3) pair interventions with evidence-based cost awareness education to drive culture change.

RESULTS: We identified 6 ongoing projects during our first year. Preliminary data for our inaugural projects are encouraging. One initiative, which targeted decreasing nebulizer use on a high-acuity medical floor (often using metereddose inhalers instead) led to a decrease in rates of more than $50 \%$.

CONCLUSIONS: The HVC program is proving to be a successful mechanism to promote improved healthcare value and clinician engagement. Journal of Hospital Medicine 2014;9:671-677. (C) 2014 Society of Hospital Medicine
With a United States medical system that spends as much as $\$ 750$ billion each year on care that does not result in improved health outcomes, ${ }^{1}$ many policy initiatives, including the Centers for Medicare and Medicaid Services' Value-Based Purchasing program, seek to realign hospitals' financial incentives from a focus on production to one on value (quality divided by cost). ${ }^{2,3}$ Professional organizations have now deemed resource stewardship an ethical responsibility for professionalism, ${ }^{4,5}$ and campaigns such as the American Board of Internal Medicine (ABIM) Foundation's Choosing Wisely effort and the American College of Physicians' High-Value Care platform are calling on frontline clinicians to address unnecessary and wasteful services. ${ }^{6,7}$

Despite these pressures and initiatives, most physicians lack the knowledge and tools necessary to prioritize the delivery of their own healthcare services according to value. ${ }^{8-10}$ Hospital medicine physicians are unaware of the costs associated with the interventions they order, ${ }^{10}$ and the majority of medical training programs lack curricula focused on healthcare costs, ${ }^{11}$ creating a large gap between physicians' per-

*Address for correspondence and reprint requests: Christopher Moriates, MD, Assistant Clinical Professor, Division of Hospital Medicine, University of California, San Francisco, 505 Parnassus Ave, M1287, San Francisco, CA 94143; Telephone: 415-476-9852; Fax: 415-502-1963; E-mail: cmoriates@medicine.ucsf.edu

Additional Supporting Information may be found in the online version of this article.

Received: February 6, 2014; Revised: June 12, 2014; Accepted: June 12, 2014

2014 Society of Hospital Medicine DOI 10.1002/jhm.2235

Published online in Wiley Online Library (Wileyonlinelibrary.com). ceived, desired, and actual knowledge related to costs. $^{12}$ Novel frameworks and frontline physician engagement are required if clinicians are to improve the value of the care they deliver.

We describe 1 of our first steps at the University of California, San Francisco (UCSF) to promote highvalue care (HVC) delivery: the creation of a HVC program led by clinicians and administrators focused on identifying and addressing wasteful practices within our hospitalist group. The program aims to (1) use financial and clinical data to identify areas with clear evidence of waste in the hospital, (2) promote evidence-based interventions that improve both quality of care and value, and (3) pair interventions with evidence-based cost awareness education to drive culture change. Our experience and inaugural projects provide a model of the key features, inherent challenges, and lessons learned, which may help inform similar efforts.

\section{METHODS}

In March 2012, we launched an HVC program within our Division of Hospital Medicine at UCSF Medical Center, a 600-bed academic medical center in an urban setting. During the 2013 academic year, our division included 45 physicians. The medicine service, comprised of 8 teaching medical ward teams (1 attending, 1 resident, 2 interns, and variable number of medical students), and 1 nonteaching medical ward team (1 attending), admitted 4700 patients that year.

\section{Organizational Framework}

The HVC program is co-led by a UCSF hospitalist (C.M.) and the administrator of the Division of 

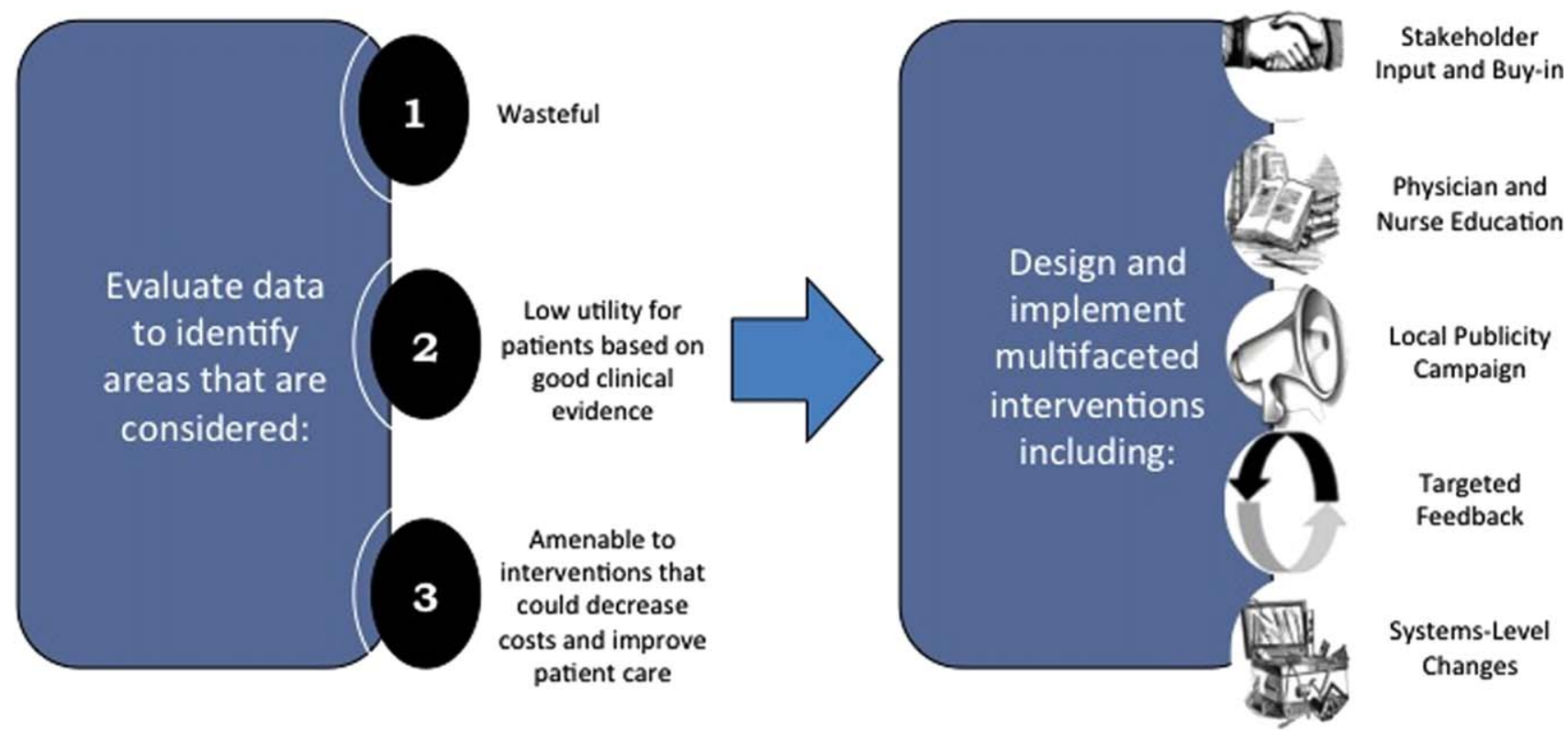

Input and Buy-in

Physician and

Nurse Education

Local Publicity

Campaign

Targeted

Feedback

Systems-Level

Changes

FIG. 1. Framework for high-value care projects.

Hospital Medicine (M.N.). Team members include hospitalists, hospital medicine fellows, resident physicians, pharmacists, project coordinators, and other administrators. The team meets in person for 1 hour every month. Project teams and ad hoc subcommittee groups often convene between meetings.

Our HVC program was placed within the infrastructure, and under the leadership, of our already established quality improvement (QI) program at UCSF. Our Division of Hospital Medicine Director of Quality and Safety (M.M.) thus oversees the QI, patient safety, patient experience, and high-value care efforts.

The HVC program funding is largely in personnel costs. The physician leader ( $15 \%$ effort) is funded by the Division of Hospital Medicine, whereas the administrator is cofunded by both the division and by the medical center (largely through her roles as both division administrator and service line director). An administrative assistant within the division is also assigned to help with administrative tasks. Some additional data gathering and project support comes from existing medical center QI infrastructure, the decision support services unit, and through UCSF's new Center for Healthcare Value. Other ancillary costs for our projects have included publicity, data analytics, and information technology infrastructure. We estimate that the costs of this program are approximately $\$ 50,000$ to $\$ 75,000$ annually.

\section{Framework for Identifying Target Projects Robust Analysis of Costs}

We created a framework for identifying, designing, and promoting projects specifically aimed at improving healthcare value (Figure 1). Financial data were used to identify areas with clear evidence of waste in the hospital, areas of high cost with no benefit in health outcomes. We focused particularly on obtaining cost and billing data for our medical service, which provided important insight into potential targets for improvements in value. For example, in 2011, the Division of Hospital Medicine spent more than \$1 million annually in direct costs for the administration of nebulized bronchodilator therapies (nebs) to nonintensive care unit patients on the medical service. ${ }^{13}$ These high costs, exposed by billing data, were believed to represent potential unnecessary testing and/or procedures. Not every area of high cost was deemed a target for intervention. For example, the use of recombinant factor VIII appeared a necessary expenditure (over $\$ 1$ million per year) for our patients with hemophilia. Although our efforts focused on reducing waste, it is worth noting that healthcare value can also be increased by improving the delivery of high-value services.

\section{Recognized Benefits in Quality of Care}

The program also evaluated the impact of cost reduction efforts on the quality of care, based on a high standard of current evidence. Though value can be improved by interventions that decrease costs while being quality neutral, our group chose to focus first on projects that would simultaneously improve quality while decreasing costs. We felt that this win-win strategy would help obtain buy-in from clinicians weary of prior cost-cutting programs. For example, we pursued interventions aimed at reducing inappropriate gastric stress ulcer prophylaxis, which had the potential to both cut costs and minimize risks of hospital-acquired pneumonia and Clostridium difficile infections. ${ }^{14,15}$ All proposed HVC targets were vetted through a review of the literature and published guidelines. In 
general, our initial projects had to be strongly supported by evidence, with high-quality studies, preferably meta-analyses or systematic reviews, that displayed the safety of our recommended changes. We reviewed the literature with experts. For example, we met with faculty pulmonologists to discuss the evidence supporting the use of inhalers instead of nebulizers in adults with obstructive pulmonary disease. The goals of our projects were chosen by our HVC committee, based on an analysis of our baseline data and the perceived potential effects of our proposed interventions.

\section{Educational Intervention}

Last, we paired interventions with evidence-based cost awareness education to drive culture change. At UCSF we have an ongoing longitudinal cost-awareness curriculum for residents, which has previously been described. ${ }^{16}$ We took advantage of this educational forum to address gaps in clinician knowledge related to the targeted areas. When launching the initiative to decrease unnecessary inpatient nebulizer usage and improve transitions to inhalers, we utilized the chronic obstructive pulmonary disease case in the costawareness series. Doing so allowed us to both review the evidence behind the effectiveness of inhalers, and introduce our Nebs No More After 24 campaign, which sought to transition adult inpatients with obstructive pulmonary symptoms from nebs to inhalers within 24 hours of admission. ${ }^{13}$

\section{Intervention Strategy}

Our general approach has been to design and implement multifaceted interventions, adapted from previous QI literature (Figure 1). ${ }^{17}$ Given the importance of frontline clinician engagement to successful project implementation, ${ }^{18-20}$ our interventions are physiciandriven and are vetted by a large group of clinicians prior to launch. The HVC program also explicitly seeks stakeholder input, perspective, and buy-in prior to implementation. For example, we involved respiratory therapists (RTs) in the design of the Nebs No More After 24 project, thus ensuring that the interventions fit within their workflow and align with their care-delivery goals.

Local publicity campaigns provide education and reminders for clinicians. Posters, such as the Nebs No More After 24 poster (Figure 2), were hung in physician, nursing, and RT work areas. Pens featuring the catchphrase Nebs No More After 24 were distributed to clinicians.

In addition to presentations to residents through the UCSF cost awareness curriculum, educational presentations were also delivered to attending physicians and to other allied members of the healthcare team (eg, nurses, RTs) during regularly scheduled staff meetings.

The metrics for each of the projects were regularly monitored, and targeted feedback was provided to clinicians. For the Nebs No More After 24 campaign, data for the number of nebs delivered on the target floor were provided to resident physicians during the cost awareness conference each month, and the data were presented to attending hospitalists in the monthly QI newsletter. This academic year, transfusion and telemetry data are presented via the same strategy.

Stakeholder recruitment, education, and promotional campaigns are important to program launches, but to sustain projects over the long-term, system changes may be necessary. We have pursued changes in the computerized provider order entry (CPOE) system, such as removing nebs from the admission order set or putting a default duration for certain telemetry orders. Systems-level interventions, although more difficult to achieve, play an important role in creating enduring changes when paired with educational interventions.

\section{RESULTS}

During our first 2 years we have initiated ongoing projects directed at 6 major targets (Table 1). Our flagship project, Nebs No More After 24, resulted in a decrease of nebulizer rates by more than $50 \%$ on a high-acuity medical floor, as previously published. ${ }^{13}$ We created a financial model that primarily accounted for RT time and pharmaceutical costs, and estimated a savings of approximately $\$ 250,000$ annually on this single medical ward (see Supporting Information, Table 1 , in the online version of this article). ${ }^{13}$

The HVC program also provided an arena for collaborating with and supporting value-based projects launched by other groups, such as the UCSF Medication Outcomes Center's inappropriate gastric stress ulcer prophylaxis program. ${ }^{21}$ Our group helped support the development and implementation of evidence-based clinical practice guidelines, and we assisted educational interventions targeting clinicians. This program resulted in a decrease in inappropriate stress ulcer prophylaxis in intensive care unit patients from $19 \%$ to $6.6 \%$ within 1 month following implementation. ${ }^{21}$

\section{DISCUSSION}

Physicians are increasingly being asked to embrace and lead efforts to improve healthcare value and reduce costs. Our program provides a framework to guide physician-led initiatives to identify and address areas of healthcare waste.

\section{Challenges and Lessons Learned Overcoming the Hurdle of More Care as Better Care}

Improving the quality of care has traditionally stressed the underuse of beneficial testing and treatments, for example the use of angiotensin-converting enzyme inhibitors in systolic heart failure. We found that improving quality by curbing overuse was a new idea 


\section{Nebs No More After 24!} Help us improve transitions from nebulizers to metered dose inhalers
(MDIs) and provide patient education about proper MDI use

$\diamond$ MDIs are as effective as nebulizer treatments!

$\diamond$ MDls provide high value, high quality patient care!

$\diamond$ We can teach and train our patients on correct MDI use while in the hospital!

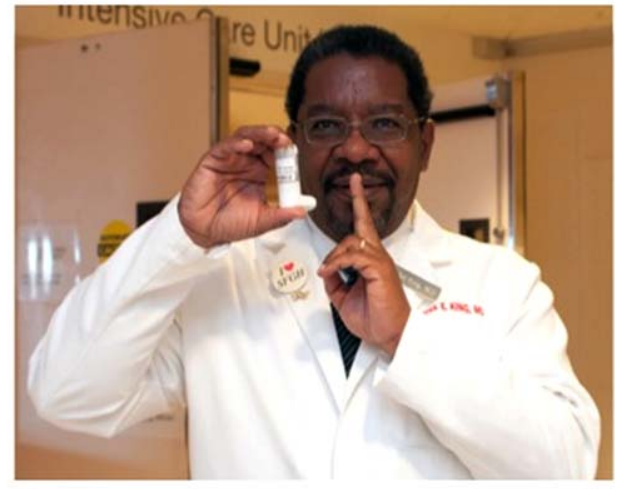

Talmadge E. King. Jr., MD Pulmonologist and Chair ot UCSF Department of Medicine -Open mouth technique: Hold MDI two tinger wiaths away from your lips."
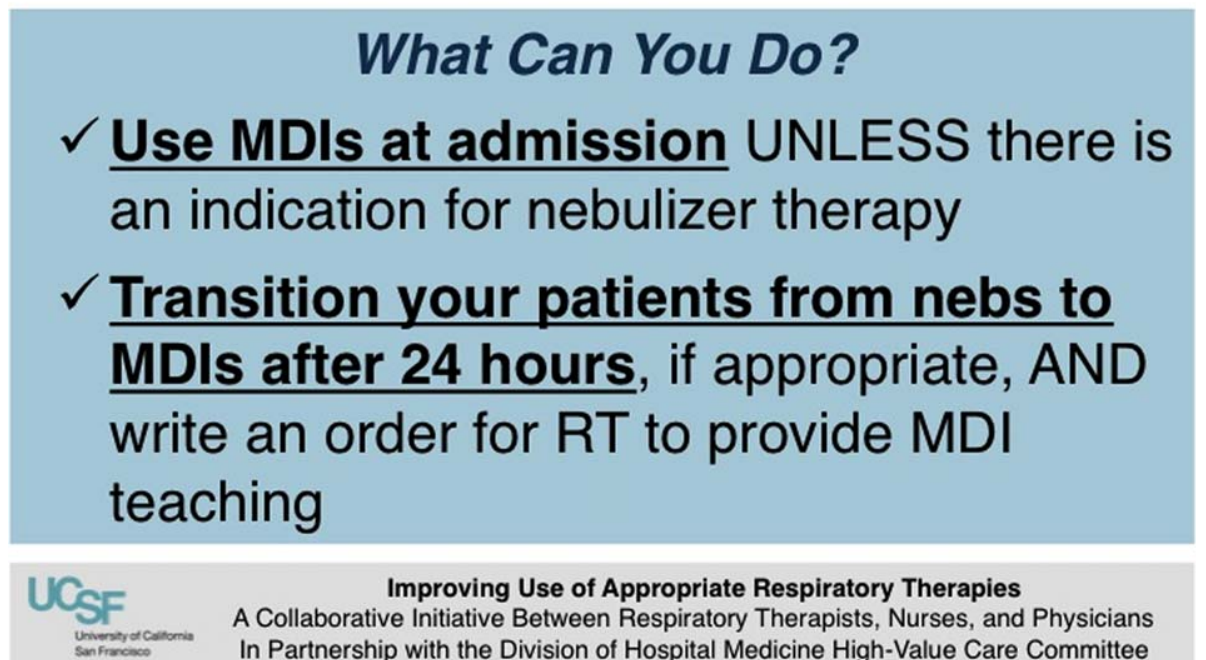

Improving Use of Appropriate Respiratory Therapies

A Collaborative Initiative Between Respiratory Therapists, Nurses, and Physicians In Partnership with the Division of Hospital Medicine High-Value Care Committee

FIG. 2. An example of a high-value care project poster.

for many physicians. Traditionally, physicians have struggled with cost reduction programs, feeling that efforts to reduce costs are indifferent to quality of care, and worse, may actually lead to inferior care. ${ }^{22}$ The historical separation of most QI and cost reduction programs has likely furthered this sentiment. Our first projects married cost reduction and QI efforts by demonstrating how reducing overuse could provide an opportunity to increase quality and reduce harms from treatments. For example, transitioning from nebs to metered-dose inhalers offered the chance to provide inpatient inhaler teaching, whereas decreasing proton pump inhibitor use can reduce the incidence of $C$ difficile. By framing these projects as addressing both numerator and denominator of the value equation, we were able to align our cost-reduction efforts with physicians' traditional notions of QI.

\section{Cost Transparency}

If physicians are to play a larger role in cost-reduction efforts, they need at least a working understanding of fixed and variable costs in healthcare and of institutional prices. $^{23,24}$ Utilization and clear information about costs were used to guide our interventions and ensured that the efforts spent to eliminate waste would result in cost savings. As an example, we learned that decreasing nebulizer use without a corresponding decrease in daily RT staffing would lead to minimal cost savings. These analyses require the support of business, financial, and resource managers in addition to physicians, nurses, project coordinators, and administrators. At many institutions the lack of price and utilization transparency presents a major barrier to the accurate analysis of cost-reduction efforts. 
TABLE 1. Initial University of California, San Francisco Division of Hospital Medicine High-Value Care Projects

\begin{tabular}{|c|c|c|c|}
\hline High-Value Care Projects & Relevant Baseline Data & Goals of Project & Strategies \\
\hline $\begin{array}{l}\text { Nebs No More After 24: Improving } \\
\text { appropriate use of } \\
\text { respiratory services }\end{array}$ & $\begin{array}{l}\text { The medicine service spent \$1 million in } \\
\text { direct costs on approximately 25,000 } \\
\text { nebs for non-ICU inpatients. }\end{array}$ & $\begin{array}{l}\text { Reduce unnecessary nebs }>15 \% \text { over } 9 \text { months. } \\
\text { Improve transitions from nebs to MDls. } \\
\text { Improve patient self-administration of MDls. }\end{array}$ & $\begin{array}{l}\text { Removed nebs from admit order set. } \\
\text { Enlisted RTs and RNs to help with MDI teaching for patients. } \\
\text { Implemented an educational program for medicine physicians. } \\
\text { Created local publicity: posters, flyers, and pens. } \\
\text { Provided data feedback to providers. } \\
\text { Next step: Introduce a CPOE--linked intervention. }\end{array}$ \\
\hline $\begin{array}{l}\text { Improving use of stress } \\
\text { ulcer prophylaxis }\end{array}$ & $\begin{array}{l}\text { 77\% of ICU patients on acid suppressive therapy; } \\
31 \% \text { of these patients did not meet criteria for } \\
\text { appropriate prophylaxis. }\end{array}$ & Reduce overuse and inappropriate use of SUP. & $\begin{array}{l}\text { A team of pharmacists, nurses, and physicians developed targeted } \\
\text { and evidence-based UCSF guidelines on use of SUP. } \\
\text { Developed and implemented a pharmacist-led intervention to } \\
\text { reduce inappropriate SUP in the ICUs that included the following: } \\
\text { Reminders on admission and discharge from ICU } \\
\text { Education and awareness initiative for prescribers } \\
\text { ICU and service champions } \\
\text { Culture change } \\
\text { Next step: Incorporate indications in CPOE and work with ICU to } \\
\text { incorporate appropriate GI prophylaxis as part of the } \\
\text { standard ICU care bundle. }\end{array}$ \\
\hline Blood utilization stewardship & $\begin{array}{l}30 \% \text { of transfusions on the hospital } \\
\text { medicine service are provided to } \\
\text { patients with a hemoglobin }>8 \mathrm{~g} / \mathrm{dL} \text {. }\end{array}$ & $\begin{array}{l}\text { Decrease units of blood transfused for a } \\
\text { hemoglobin }>8.0 \mathrm{~g} / \mathrm{dL} \text { by } 25 \% \text {. }\end{array}$ & $\begin{array}{l}\text { Launched an educational campaign for attending and } \\
\text { resident physicians. } \\
\text { Monthly feedback to residents and attending physicians. } \\
\text { Next step: Introduce a decision support system in the CPOE for } \\
\text { blood transfusion orders in patients with most recent } \\
\text { hemoglobin level }>8 \text {. }\end{array}$ \\
\hline Improving telemetry utilization & $\begin{array}{l}\text { 44\% of monitored inpatients on the medical service } \\
\text { (with length of stay }>48 \text { hours) remain on } \\
\text { telemetry until discharge. }\end{array}$ & $\begin{array}{l}\text { Decrease by } 15 \% \text { the number of patients } \\
\text { (with length of stay }>48 \text { hours) who } \\
\text { remain on telemetry until discharge. }\end{array}$ & $\begin{array}{l}\text { Implemented an educational campaign for nursing groups and } \\
\text { the medicine and cardiology housestaff. } \\
\text { Launched a messaging campaign consisting of posters and } \\
\text { pocket cards on appropriate telemetry use. } \\
\text { Designed a feedback campaign with monthly e-mail to housestaff } \\
\text { on their ward team's telemetry use stats. } \\
\text { Next step: Build a CPOE intervention that asks users to specify } \\
\text { an approved indication for telemetry when they order } \\
\text { monitoring. The indication then dictates how long the order } \\
\text { is active (24, } 48,72 \text { hours or ongoing), and the MD must } \\
\text { renew the order after the elapsed time. }\end{array}$ \\
\hline $\begin{array}{l}\text { iReduce iCal: ordering ionized } \\
\text { calcium only when needed }\end{array}$ & $\begin{array}{l}\text { The medicine service spent } \$ 167,000 \text { in direct costs } \\
\text { on iCal labs over a year ( } 40 \% \text { of all calcium lab } \\
\text { orders; } 42 \% \text { occurred in non-ICU patients). }\end{array}$ & $\begin{array}{l}\text { Reduce number of iCal labs drawn on the } \\
\text { medicine service by }>25 \% \text { over the } \\
\text { course of } 6 \text { months. }\end{array}$ & $\begin{array}{l}\text { With the introduction of CPOE, iCal was removed from } \\
\quad \text { traditional daily lab order sets. } \\
\text { Discussed with lab, renal, and ICU stakeholders. } \\
\text { Implemented an educational campaign for physicians and nurses. } \\
\text { Created local publicity: posters and candies. } \\
\text { Provided data feedback to providers. }\end{array}$ \\
\hline $\begin{array}{l}\text { Repeat inpatient } \\
\text { echocardiograms }\end{array}$ & $\begin{array}{l}25 \% \text { of TTEs are performed within } 6 \text { months } \\
\text { of a prior; one-third of these are for } \\
\text { inappropriate indications. }\end{array}$ & Decrease inappropriate repeat TTEs by $25 \%$. & $\begin{array}{l}\text { Implemented an educational campaign. } \\
\text { Next step: provide the most recent TTE results in the CPOE at } \\
\text { time of order, and provide auditing and decision } \\
\text { support for repeat TTEs. }\end{array}$ \\
\hline
\end{tabular}

NOTE: Abbreviations: CPOE, computerized provider order entry; Gl, gastrointestinal; iCal, ionized calcium; ICU, intensive care unit; MD, medical doctor; MDls, metered-dose inhalers; nebs, nebulized bronchodilator treatment; $\mathrm{RN}$, registered nurse; RT, respiratory therapist; SUP, stress ulcer prophylaxis; $\Pi \pi E$, transthoracic echocardiogram; UCSF, University of California, San Francisco.

\section{The Diplomacy of Cost-Reduction}

Because the bulk of healthcare costs go to labor, efforts to reduce cost may lead to reductions in the resources available to certain departments or even to individuals' wages. For example, initiatives aimed at reducing inappropriate diagnostic imaging will affect the radiology department, which is partially paid based on the volume of studies performed. ${ }^{25}$ Key stakeholders must be identified early, and project leaders should seek understanding, engagement, and buy-in from involved parties prior to implementation. There will often be times that support from senior leaders will be needed to negotiate these tricky situations.

Although we benefited from a largely supportive hospital medicine faculty and resident physicians, not all of our proposed projects made it to implementation. Sometimes stakeholder recruitment proved to be difficult. For instance, a proposed project to change the protocol from routine to clinically indicated peripheral intravenous catheter replacement for adult inpatients was met with some resistance by some members of nursing management. We reviewed the literature together and discussed in length the proposal, but ultimately decided that our institution was not ready for this change at this time.

\section{Limitations and Next Steps}

Our goal is to provide guidance on exporting the approach of our HVC program to other institutions, but there may be several limitations. First, our 
strategy relied on several contributing factors that may be unique to our institution. We had engaged frontline physician champions, who may not be available or have the necessary support at other academic or community organizations. Our UCSF cost awareness curriculum provided an educational foundation and framework for our projects. We also had institutional commitment in the form of our medical center division administrator.

Second, there are up-front costs to running our committee, which are primarily related to personnel funding as described in the Methods. Over the next year we aim to calculate cost-effectiveness ratios for our projects and overall return on investment for each of our projects, as we have done for the Nebs No More After 24 project (see Supporting Information, Table 1 , in the online version of this article). Based on this analysis, the modest upfront costs appear to be easily recouped over the course of the year.

We have anecdotally noted a culture change in the way that our physicians discuss and consider testing. For example, it is common now to hear ward teams on morning rounds consider the costs of testing or discuss the need for prophylactic proton pump inhibitors. An important next step for our HVC program is the building of better data infrastructures for our own electronic health record system to allow us to more quickly, accurately, and comprehensively identify new targets and monitor the progress and sustainability of our projects. The Institute of Medicine has noted that the adoption of technology is a key strategy to creating a continuously learning healthcare system. ${ }^{1}$ It is our hope that through consistent audit and feedback of resource utilization we can translate our early gains into sustainable changes in practice.

Furthermore, we hope to target and enact additional organizational changes, including creating CPOE-linked interventions to help reinforce and further our objectives. We believe that creating systems that make it easier to do the right thing will help the cause of embedding HVC practices throughout our medical center. We have begun to scale some of our projects, such as the Nebs No More After 24 campaign, medical center wide, and ultimately we hope to disseminate successful projects and models beyond our medical center to contribute to the national movement to provide the best care at lower costs.

As discussed above, our interventions are targeted at simultaneous improvements in quality with decreased costs. However, the goal is not to hide our cost interventions behind the banner of quality. We believe that there is a shifting culture that is increasingly ready to accept cost alone as a meaningful patient harm, worthy of interventions on its own merits, assuming that quality and safety remain stable. 26,27

\section{CONCLUSIONS}

Our HVC program has been successful in promoting improved healthcare value and engaging clinicians in this effort. The program is guided by the use of financial data to identify areas with clear evidence of waste in the hospital, the creation of evidence-based interventions that improve quality of care while cutting costs, and the pairing of interventions with evidence-based cost awareness education to drive culture change.

\section{Acknowledgements}

The authors acknowledge the following members of the UCSF Division of Hospital Medicine High-Value Care Committee who have led some of the initiatives mentioned in this article and have directly contributed to Table 1: Dr. Stephanie Rennke, Dr. Alvin Rajkomar, Dr. Nader Najafi, Dr. Steven Ludwin, and Dr. Elizabeth Stewart. Dr. Russ Cucina particularly contributed to the designs and implementation of electronic medical record interventions.

Disclosures: Dr. Moriates received funding from the UCSF Center for Healthcare Value, the Agency for Healthcare Research and Quality (as editor for AHRQ Patient Safety Net), and the ABIM Foundation. Mrs. Novelero received funding from the UCSF Center for Healthcare Value. Dr. Wachter reports serving as the immediate past-chair of the American Board of Internal Medicine (for which he received a stipend) and is a current member of the ABIM Foundation board; receiving a contract to UCSF from the Agency for Healthcare Research and Quality for editing 2 patient-safety websites; receiving compensation from John Wiley \& Sons for writing a blog; receiving compensation from QuantiaMD for editing and presenting patient safety educational modules; receiving royalties from Lippincott Williams \& Wilkins and McGraw-Hill for writing/editing several books; receiving a stipend and stock/options for serving on the Board of Directors of IPC-The Hospitalist Company; serving on the scientific advisory boards for PatientSafe Solutions, CRISI, SmartDose, and EarlySense (for which he receives stock options); and holding the Benioff endowed chair in hospital medicine from Marc and Lynne Benioff. He is also a member of the Board of Directors of Salem Hospital, Salem, Oregon, for which he receives travel reimbursement but no compensation. Mr. John Hillman, Mr. Aseem Bharti, and Ms. Claudia Hermann from UCSF Decision Support Services provided financial data support and analyses, and the UCSF Center for Healthcare Value provided resource and financial support.

\section{References}

1. Institute of Medicine. Committee on the Learning Health Care System in America. Best Care at Lower Cost: The Path to Continuously Learning Health Care in America. Washington, DC: National Academies Press; 2012.

2. VanLare J, Conway P. Value-based purchasing-national programs to move from volume to value. N Engl J Med. 2012;367(4):292-295.

3. Berwick DM. Making good on ACOs' promise-the final rule for the Medicare Shared Savings Program. N Engl J Med. 2011;365(19): 1753-1756.

4. Snyder L. American College of Physicians ethics manual: sixth edition. Ann Intern Med. 2012;156(1 pt 2):73-104.

5. ABIM Foundation, American College of Physicians-American Society of Internal Medicine, European Federation of Internal Medicine. Medical professionalism in the new millennium: a physician charter. Ann Intern Med. 2002;136(3):243-246.

6. Cassel CK, Guest JA. Choosing Wisely: helping physicians and patients make smart decisions about their care. JAMA. 2012;307(17): 1801.

7. Owens DK, Qaseem A, Chou R, Shekelle P. High-value, costconscious health care: concepts for clinicians to evaluate the benefits, harms, and costs of medical interventions. Ann Intern Med. 2011; 154(3):174-180.

8. Chien AT, Rosenthal MB. Waste not, want not: promoting efficient use of health care resources. Ann Intern Med. 2013;158(1):67-68.

9. Rock TA, Xiao R, Fieldston E. General pediatric attending physicians' and residents' knowledge of inpatient hospital finances. Pediatrics. 2013;131(6):1072-1080.

10. Graham JD, Potyk D, Raimi E. Hospitalists' awareness of patient charges associated with inpatient care. J Hosp Med. 2010;5(5):295297.

11. Patel MS, Reed DA, Loertscher L, McDonald FS, Arora VM. Teaching residents to provide cost-conscious care: A national survey of residency program directors. JAMA Intern Med. 2014;174(3):470-472.

12. Adiga K, Buss M, Beasley BW. Perceived, actual, and desired knowledge regarding medicare billing and reimbursement. J Gen Intern Med. 2006;21(5):466-470.

13. Moriates C, Novelero M, Quinn K, Khanna R, Mourad M. "Nebs No More After 24": a pilot program to improve the use of appropriate respiratory therapies. JAMA Intern Med. 2013;173(17): 1647-1648. 
14. Herzig SJ, Howell MD, Ngo LH, Marcantonio ER. Acid-suppressive medication use and the risk for hospital-acquired pneumonia. JAMA. 2009;301(20):2120-2128.

15. Howell MD, Novack V, Grgurich P, et al. Iatrogenic gastric acid suppression and the risk of nosocomial Clostridium difficile infection. Arch Intern Med. 2010;170(9):784-790.

16. Moriates C, Soni K, Lai A, Ranji S. The value in the evidence: teaching residents to "choose wisely." JAMA Intern Med. 2013;173(4):308-310.

17. Shojania KG, Grimshaw JM. Evidence-based quality improvement: the state of the science. Health Aff. 2005;24(1):138-150.

18. Caverzagie KJ, Bernabeo EC, Reddy SG, Holmboe ES. The role of physician engagement on the impact of the hospital-based practice improvement module (PIM). J Hosp Med. 2009;4(8):466-470.

19. Gosfield AG, Reinertsen JL. Finding common cause in quality: confronting the physician engagement challenge. Physician Exec. 2008; $34(2): 26-28,30-31$.

20. Conway PH, Cassel CK. Engaging physicians and leveraging professionalism: a key to success for quality measurement and improvement. JAMA. 2012;308(10):979-980.
21. Leon N de, Sharpton S, Burg C, et al. The development and implementation of a bundled quality improvement initiative to reduce inappropriate stress ulcer prophylaxis. ICU Dir. 2013;4(6): $322-325$.

22. Beckman HB. Lost in translation: physicians' struggle with costreduction programs. Ann Intern Med. 2011;154(6):430-433.

23. Kaplan RS, Porter ME. How to solve the cost crisis in health care. Harv Bus Rev. 2011;89(9):46-52, 54, 56-61 passim.

24. Rauh SS, Wadsworth EB, Weeks WB, Weinstein JN. The savings illusion-why clinical quality improvement fails to deliver bottom-line results. N Engl J Med. 2011;365(26):e48.

25. Neeman N, Quinn K, Soni K, Mourad M, Sehgal NL. Reducing radiology use on an inpatient medical service: choosing wisely. Arch Intern Med. 2012;172(20):1606-1608

26. Moriates C, Shah NT, Arora VM. First, do no (financial) harm. JAMA. 2013;310(6):577-578.

27. Ubel PA, Abernethy AP, Zafar SY. Full disclosure-out-ofpocket costs as side effects. N Engl J Med. 2013;369(16): 1484-1486. 